\title{
Research Article \\ Receding Horizon Unbiased FIR Filters and Their Application to Sea Target Tracking
}

\author{
Boris Skorohod \\ Department of Informatics and Control in Technical Systems, Sevastopol State University, 33 Universitetskaya Street, \\ Sevastopol 299053, Russia \\ Correspondence should be addressed to Boris Skorohod; boris.skorohod@mail.ru
}

Received 14 August 2018; Revised 28 October 2018; Accepted 18 November 2018; Published 16 December 2018

Academic Editor: Ai-Guo Wu

Copyright (C) 2018 Boris Skorohod. This is an open access article distributed under the Creative Commons Attribution License, which permits unrestricted use, distribution, and reproduction in any medium, provided the original work is properly cited.

Finite impulse response (FIR) state estimation algorithms have been much discussed in literature lately. It is well known that they allow overcoming the Kalman filter divergence caused by modeling uncertainties. In this paper, new receding horizon unbiased FIR filters ignoring noise statistics for time-varying discrete state-space models are proposed. They have the following advantages. First, the proposed filters use only known means of state vector components at starting points of sliding windows. This allows us to take into account priory statistical information (on average) about specified movements of the system. Second, the iterative version of the filter has a Kalman-like form. Besides, its initialization does not include a training cycle in a batch form. Such filters may have a wide range of applications. In this paper, position and speed estimation of sea targets using angle measurements in azimuth and elevation is considered as an example.

\section{Introduction}

Finite impulse response (FIR) state estimation algorithms can be considered as an alternative to the Kalman filter (KF). These algorithms allow us to overcome its divergence caused by model inaccuracy (incorrectly chosen models, temporary perturbations, and errors in the setting of the noise statistics) [1-3]. Divergence prevention is achieved by reducing the impact of the data or rejection the data out of the sliding window. In this context, the $\mathrm{KF}$ can be considered as an estimation algorithm with infinite impulse response (IIR).

Quite a number of methods have been offered to construct FIR algorithms. In [4], the FIR filter for a linear homogeneous system is proposed. The optimal unbiased FIR (OUFIR) and receding horizon algorithms are proposed in [2, $3,5-7]$. Initial conditions at starting points of sliding windows are assumed to be diffuse random variables or unknown arbitrary values. In [7], the receding horizon optimal unbiased FIR (RHOUFIR) filter which obtains information about the window initial conditions from finite observations is suggested. Another approach ensuring optimality and unbiasedness in a finite number of steps is described in $[8,9]$. The RHOUFIR filter suggested by the authors uses known statistical information for parts of state vector components at starting points of sliding windows. Within the framework of covariance analysis this allows us to take into account priory statistical information about random biases, trends, and specified movements of the system. Training tasks of neural and neurofuzzy networks are another example of possible application of this filter [9].

In [10-14], the unbiased FIR (UFIR) filters ignoring the noise statistics of the process, measurements, and the statistics of state vector initial conditions are developed. Computer modeling shows that the UFIR filters can be more robust than the KF or the OUFIR filter if the size of the sliding window is properly fitted. There are several general approaches to select $N$ based on a heuristic choice, via the mean square errors, via real measurements, and via the use of bandlimited property of signals [13]. However, the proposed recursive filters have two disadvantages. First, a butch form of the algorithm is needed for the initialization of the recursive UFIR filter during the learning cycle which may be inconvenient or even problematic in some cases (e.g., in case of gaps in the observations, estimation parameters of nonlinear systems, and nonstationary processes). Second, these filters do not use 
priorly known information for initialization at starting points of sliding windows.

In this paper, the receding horizon UFIR (RHUFIR) filters using only known means of state vector components at starting points of sliding windows are suggested. They allow us to take into account (on average) priory statistical information about the system. Furthermore, for the iterative filter initialization no training cycle in a batch form is required. While the implementation of the RHOUFIR filter described in $[8,9]$ may be difficult due to the lack of sufficiently accurate statistical information about system, the algorithms under consideration may produce quite acceptable estimates. Theoretically this can be justified by a selection of the optimal or sub-optimal values of $N$ using the algorithms described in [13]. Another important feature is that the RHUFIR filter is computationally less demanding in comparison with the RHOUFIR. This is important, for example, in the tasks of neural and neurofuzzy networks training.

As a possible application of the proposed filters, the position and speed estimation of sea targets using angle measurements in azimuth and elevation from the observership's video camera is considered. The general approach to solve this problem is to use Kalman filtering methods [15]. The difficulties arising due to the specificity of both the problem itself and the methods commonly used are well known. First, measurement models may be highly nonlinear near the state estimate [16]. Second, the object state may not be observable if the observer does not maneuver in a special way $[17,18]$. Third, there is a large initial uncertainty about the position, speed, and acceleration of the observed object. All this can lead to a divergence of the KF and its various modifications [19-21].

The work is organized as follows. The RHUFIR state estimation problem is formulated in Section 2. Sections 3 and 4 concern the batch and iterative RHUFIR filters, respectively. Section 5 contains a comparative analysis of the RHUFIR and RHOUFIR filters. A diffuse approach to FIR estimation and its connection with the RHOUFIR filter is considered in Section 6. The position and speed estimation of sea objects is considered in Section 7. The conclusions are presented in Section 8.

\section{Problem Statement}

Let us consider a linear discrete time-variant system of the form

$$
\begin{aligned}
x_{t+1} & =A_{t} x_{t}+B_{t} w_{t}, \\
y_{t} & =C_{t} x_{t}+D_{t} \xi_{t},
\end{aligned}
$$

where $x_{t} \in R^{n}$ is the state vector, $y_{t} \in R^{m}$ is the measured output vector, $w_{t} \in R^{r}$ and $\xi_{t} \in R^{l}$ are random processes with zero means, i.e., $E\left(w_{t}\right)=0, E\left(\xi_{t}\right)=0, A_{t}, B_{t}, C_{t}, D_{t}$ are known matrices of appropriate dimensions, $t \in\{0,1, \ldots\}$.

Consider the discrete intervals $[t-N, t-1], t \in T_{N}=$ $\{N, N+1, \ldots\}$ (the sliding windows), where $N$ is the horizon length. Let us assume that the following conditions are met.

A1: Means for arbitrary $q \in\{1,2, \ldots, n-1\}$ components of the state vector at the starting points $x_{t-N}$ of the sliding windows are known. A priori information on remaining $n-q$ components of $x_{t-N}, t \in T_{N}$ is absent and they are either unknown constants or random variables statistical characteristics of which are unknown. The value $q=0$ is used further if a priori information about all components of $x_{t-N}$ is absent.

A2: If $q \neq 0$ then without loss of generality it is assumed that the state vector elements are arranged so that means $m_{i, t-N}=E\left(x_{i, t-N}\right), i=1,2, \ldots, q$, are known, where $x_{t}=$ $\left(x_{1, t}, \ldots, x_{q, t}, x_{q+1, t}, \ldots, x_{n, t}\right)^{T}, t \in T_{N}=\{N, N+1, \ldots\}$.

It is required to find the linear state estimate of the convolution-based batch form

$$
\widehat{x}_{t}=\Omega_{t, t-N} m_{t-N}+\sum_{i=t-N}^{t-1} Y_{t, i} y_{i}, \quad t \in T_{N}
$$

from measurements $y_{t-N}, y_{t-N+1}, \ldots, y_{t-1}$ and its recursive representation for $\widehat{x}_{t}$ calculation, where the matrices $Y_{t, i} \in$ $R^{n \times m}$ and $\Omega_{t, t-N} \in R^{n \times q}$ do not depend on a priori information about the unknown vector $x_{t-N}, m_{t-N}=$ $\left(m_{1, t-N}, m_{2, t-N} \ldots, m_{q, t-N}\right)^{T}, q \in\{1,2, \ldots, n-1\}$, and $\Omega_{t, t-N}=$ $0, m_{t-N}=0$ if $q=0$. The estimate is found under the conditions that it is unbiased

$$
E\left(\widehat{x}_{t}\right)=E\left(x_{t}\right), \quad t \in T_{N} .
$$

The first component in (2) allows to take into account (on average) a priori information about the system (1) at starting points of sliding windows $x_{t-N}, t \in T_{N}$. This may be necessary if the pair $\left\{A_{t}, C_{t}\right\}$ is not observable but the estimate must be unbiased after processing the finite number of observations. Typical examples are models including random biases, trends and specified movements of the system (see the example in Section 7.3). The relation $m_{i, t-N}=$ const, $t \in T_{N}, i=$ $1,2, \ldots, q$, is the simplest case describing a random bias with a known mean. Note that the inference of the UFIR filter in [11] for $q=0$ is based on the assumption that the pair $\left\{A_{t}, C_{t}\right\}$ is observable. Note also that this assumption is not required for the KF. The need for the first component in (3) may also arise in the tasks of neural and neurofuzzy networks training which have a separable structure [22]. It is assumed that the linearly incoming parameters are unknown and in addition to the training set a priori information only about the nonlinearly incoming parameters is given [9]. This information is obtained from the distribution of a generating sample, a training set, or some linguistic information. The RHUFIR filter is used to estimate the parameters by linearizing networks relations in the vicinity of the last estimate.

\section{Batch Receding Horizon UFIR Filter}

The RHUFIR filter in a butch form is specified by the following theorem.

Theorem 1. Let the condition

$$
M_{t}=\sum_{i=t-N}^{t-1} R_{i}^{T} C_{i}^{T} C_{i} R_{i}>0, \quad t \in T_{N}
$$

be fulfilled. Then the unbiased estimate of the system state (1) in the batch form is determined by the expression (2), where

$$
Y_{t, i}=R_{t} M_{t}^{-1} R_{i}^{T} C_{i}^{T}+\Theta_{t, i}^{T}
$$




$$
\begin{aligned}
& \Omega_{t, t-N} \\
& = \begin{cases}Z_{t-N, t}^{T}\left(e_{1}, e_{2}, \ldots, e_{q}\right), & q \in\{1,2, \ldots, n-1\} \\
0, & q=0,\end{cases} \\
& Z_{t-N, t}=\Phi_{t, t-N}^{T}-\sum_{i=t-N}^{t-1} \Phi_{i, t-N}^{T} Y_{i, t-N}^{T} \\
& \Phi_{t+1, t-N}=A_{t} \Phi_{t, t-N}, \Phi_{t-\mathrm{N}, t-N}=I_{n} \\
& R_{i}=\Phi_{i, t-N} \Lambda_{q} \text {, } \\
& \left(\Theta_{t-N, t}, \Theta_{t-N+1, t}, \ldots, \Theta_{t-1, t}\right)=\left(I_{N(n-q)}-\Omega^{+} \Omega\right) \Xi, \\
& \Lambda_{q}=\left(e_{q+1}, e_{q+2}, \ldots, e_{n}\right), \quad q \in\{0,1, \ldots, n-1\},
\end{aligned}
$$

$\Omega=\left(R_{t-N}^{T} C_{t-N}^{T}, R_{t-N+1}^{T} C_{t-N+1}^{T}, \ldots, R_{t-1}^{T} C_{t-1}^{T}\right) \in R^{(n-q) \times N m}, \Xi$ is arbitrary $(n-q) N \times(n-q) N$ matrix, $\Omega^{+}$is the Moore Penrose inversion of $\Omega, e_{i} \in R^{n}$ is ith unit vector, and $I_{n}$ is the identity matrix of the size $n$.

Proof. First, we show that the estimation problem is equivalent to a dual control problem. Consider an auxiliary linear system of the form

$$
\begin{aligned}
& Z_{i, t}=A_{i}^{T} Z_{i+1, t}-C_{i}^{T} U_{i, t}, \\
& Z_{t, t}=I_{n},
\end{aligned}
$$

$$
i \in[t-N, t-1], t \in T_{N}
$$

where $Z_{i, t} \in R^{n \times n}$ is the state matric and $U_{i, t} \in R^{m \times n}$ is a control. Suppose that there is a control $U_{i, t}$ bringing the system (12) in a state satisfying the condition $\Lambda_{q}^{T} Z_{t-N, t}=0$ for any $t \in T_{N}$. Let us show that then there is an unbiased state estimate of the system (1) of the form (2), where

$$
Y_{t, i}=U_{i, t}^{T}
$$

and $\Omega_{t, t-N}$ is determined by (6).

Using the identity

$$
x_{t}=Z_{t-N, t}^{T} x_{t-N}+\sum_{i=t-N}^{t-1}\left(Z_{i+1, t}^{T} x_{i+1}-Z_{i, t}^{T} x_{i}\right),
$$

(1) and (12) give

$$
x_{t}=Z_{t-N, t}^{T} x_{t-N}+\sum_{i=t-N}^{t-1}\left(Z_{i+1, t}^{T} B_{i} w_{i}+U_{i, t}^{T} C_{i} x_{i}\right) .
$$

Taking into account this expression, the estimation error at the moment $t$ can be presented in the form

$$
\begin{aligned}
e_{t} & =x_{t}-\hat{x}_{t} \\
& =Z_{t-N, t}^{T}\left(q_{1}+q_{2}\right)+\sum_{i=t-N}^{t-1}\left(Z_{i+1, t}^{T} B_{i} w_{i}-U_{i, t}^{T} D_{i} \xi_{i}\right),
\end{aligned}
$$

where

$$
\begin{aligned}
& q_{1}= \begin{cases}\left(h_{1}, h_{2}, \ldots, h_{q}, 0_{1 \times(n-q)}\right)^{T}, & q \in\{1,2, \ldots, n-1\} \in R^{n}, \\
0, & q=0\end{cases} \\
& q_{2}= \begin{cases}\left(0_{1 \times q}, x_{q+1, t-N}, x_{q+2, t-N}, \ldots, x_{n, t-N}\right)^{T}, & q \in\{1,2, \ldots, n-1\} \in R^{n}, \\
x_{t-N}, & q=0\end{cases}
\end{aligned}
$$

$h_{i}=x_{i, t-N}-m_{i, t-N}, i=1,2, \ldots, q$, and $0_{1 \times q}$ is $1 \times q$ the vector row with zero elements.

Averaging the left and right sides of this expression and using the condition $\Lambda_{q}^{T} Z_{t-N, t}=0$ get

$$
\begin{gathered}
E\left(e_{t}\right)=Z_{t-N, t}^{T} E\left(q_{2}\right)=Z_{t-N, t}^{T} \Lambda_{q}\left[E\left(x_{q+1, t-N}\right),\right. \\
\left.E\left(x_{q+2, t-N}\right), \ldots, E\left(x_{n, t-N}\right)\right]^{T}=0 .
\end{gathered}
$$

Now we find the control providing a solution to the dual problem. Iterating (12) gives

$$
Z_{i, t}=G_{i, t} Z_{t, t}-\sum_{j=i}^{t-1} G_{i, j} C_{j}^{T} U_{j, t},
$$

where the matrix $G_{i, s}$ is determined by the system

$$
\begin{aligned}
& G_{i, \mathrm{~s}}=A_{i}^{T} G_{i+1, s}, \\
& G_{s, s}=I_{n},
\end{aligned}
$$

$$
s \geq i
$$

Using the boundary condition $\Lambda^{T} Z_{t-N, t}=0$, we obtain with help of (19) the linear equations system

$$
0=\Lambda_{q}^{T} G_{t-N, t} Z_{t, t}-\sum_{j=t-N}^{t-1} \Lambda_{q}^{T} G_{t-N, j} C_{j}^{T} U_{j, t} .
$$

We find a solution of this system in the form

$$
U_{j, t}=C_{j} G_{t-N, j}^{T} \Lambda_{q} L+\Omega_{j, t},
$$


where $L \in R^{(n-q) \times n}$ is a constant unknown matrix and $\Theta_{j, t}$ is arbitrary $m \times n$ matric function satisfying the condition

$$
\sum_{j=t-N}^{t-1} \Lambda_{q}^{T} G_{t-N, j} C_{j}^{T} \Theta_{j, t}=0 .
$$

Substituting (22) into (21) and taking in account (23) gives $L=W_{t}^{-1} \Lambda_{q}^{T} G_{t-N, t} Z_{t, t}$ provided $W_{t}>0$, where

$$
W_{t}=\sum_{j=t-N}^{t-1} \Lambda_{q}^{T} G_{t-N, j} C_{j}^{T} C_{j} G_{t-N, j}^{T} \Lambda_{q} .
$$

Using the expressions

$$
\begin{aligned}
G_{j, t} & =A_{j}^{T} A_{j+1}^{T} \ldots A_{t-2}^{T} A_{t-1}^{T}, \\
\Phi_{t, j} & =A_{t-1} A_{t-2} \cdots A_{j-1} A_{j}, \\
G_{j, t}^{T} & =\Phi_{t, j}, \\
M_{t} & =W_{t},
\end{aligned}
$$

we obtain

$$
\begin{aligned}
U_{j, t} & =C_{j} G_{t-N, j}^{T} \Lambda_{q} W_{t}^{-1} \Lambda_{q}^{T} G_{t-N, j} Z_{t, t}+\Theta_{j, t} \\
& =C_{j} \Phi_{j, t-N} \Lambda_{q} M_{t}^{-1} \Lambda_{q}^{T} \Phi_{t, t-N}^{T} Z_{t, t}+\Theta_{j, t} \\
& =C_{j} R_{j} M_{t}^{-1} R_{t}^{T} Z_{t, t}+\Theta_{j, t} .
\end{aligned}
$$

But as $Z_{t, t}=I_{n}$ and (10) is the general solution of the linear homogeneous system (23) then it implies (5) and (6).

Comment 1. The condition $\left\{A_{i}, C_{i}\right\}$ is observable for $i \in\{t-$ $N, t-N+1, \ldots, t-1\}$, and $t \in T_{N}=\{N, N+1, \ldots\}$ implies (3).

Comment 2. The filter takes a particularly simple form for $q=$ 0 and $\Theta_{t, i}=0$. We find from Theorem 1 that

$$
\widehat{x}_{t}=\Phi_{t, t-N} M_{t}^{-1} \sum_{i=t-N}^{t-1} \Phi_{i, t-N}^{T} C_{i}^{T} y_{i}, \quad t \in T_{N},
$$

where

$$
M_{t}=\sum_{i=t-N}^{t-1} \Phi_{i, t-N}^{T} C_{i}^{T} C_{i} \Phi_{i, t-N}>0 .
$$

These relations are equal to the algorithm from [11] up to the notation used if to ignore the one-step prediction.

\section{Iterative Form of Batch Receding Horizon UFIR Filter}

We will need the following assertion.

Lemma 2. Let the condition (4) be true. Then the control bringing the system (12) in a state satisfying the condition $\Lambda_{q}^{T} Z_{t-N, t}=0$ can be presented in the form

$$
U_{i, t}^{f}=K_{i}^{T} Z_{i+1, t}^{f}, \quad i \in[t-N, t-1], t \in T_{N},
$$

where

$$
\begin{aligned}
K_{i} & =R_{i+1} M_{i+1}^{+} R_{i}^{T} C_{i}^{T}, \\
Z_{i, t}^{f} & =\left(A_{i}^{T}-C_{i}^{T} K_{i}^{T}\right) Z_{i+1, t}^{f}, \\
Z_{t, t}^{f} & =I_{n},
\end{aligned}
$$

$$
i \in[t-N, t-1], t \in T_{N} .
$$

Proof. Let us show that the control (29) indeed solves our problem. Consider the system (12) under the action of the controls (22) with $\Theta_{j, t}=0$. We show that $Z_{i, t}=Z_{i, t}^{f}$. Substituting $U_{i, t}$ with $\Theta_{i, t}=0$ in (12) gives

$$
\begin{aligned}
& Z_{i, t}=A_{i}^{T} Z_{i+1, t}-C_{i}^{T} C_{i} R_{i} M_{t}^{-1} R_{t}^{T}, \\
& \begin{array}{l}
Z_{t, t}=I_{n}, \\
\quad i \in[t-N, t-1], t \in T_{N} .
\end{array}
\end{aligned}
$$

From comparison of right parts of (31) and (32), it follows that the solutions of the systems really coincide if $U_{i, t}^{f}=U_{i, t}$, i.e.,

$$
K_{i}^{T} Z_{i+1, t}=C_{i} R_{i} M_{t}^{-1} R_{t}^{T} .
$$

Iterating the system (32), we successively find

$$
\begin{aligned}
& Z_{i, t}=\Phi_{t, i}^{T}-\sum_{j=i}^{t-1} \Phi_{j, i}^{T} C_{j}^{T} C_{j} R_{j} M_{t}^{-1} R_{t}^{T}, \\
& K_{i}^{T} Z_{i+1, t}=C_{i} R_{i} M_{i+1}^{+} R_{i+1}^{T}\left(\Phi_{t, i+1}^{T}\right. \\
& \left.-\sum_{j=i+1}^{t-1} \Phi_{j, i+1}^{T} C_{j}^{T} C_{j} R_{j} M_{t}^{-1} R_{t}^{T}\right)=C_{i} R_{i} M_{i+1}^{+}\left(I_{n}\right. \\
& \left.-\sum_{j=i+1}^{t-1} R_{j}^{T} C_{j}^{T} C_{j} R_{j} M_{t}^{-1}\right) R_{t}^{T} \\
& =C_{i} R_{i} M_{i+1}^{+} M_{i+1} M_{t}^{-1} R_{t}^{T} .
\end{aligned}
$$

The expressions (34) and (35) follow from the identities

$$
\begin{aligned}
R_{i+1} & =\Phi_{i+1, t-N} \Lambda_{q}, \\
\Phi_{t, i+1} \Phi_{i+1, t-N} & =\Phi_{t, t-N}, \\
\Phi_{j, i+1} R_{i+1} & =\Phi_{j, i+1} \Phi_{i+1, t-N} \Lambda_{q}=\Phi_{j, t-N} \Lambda_{q}=R_{j} .
\end{aligned}
$$

Let us transform (35) using the orthogonal decomposition $M_{i+1}=V_{i} V_{i}^{T}$, where

$$
\begin{aligned}
V_{i} & =\left(R_{t-N}^{T} C_{t-N}^{T}, R_{t-N+1}^{T} C_{t-N+1}^{T} \ldots, R_{i}^{T} C_{i}^{T}\right) \\
& \left.\in R^{(n-q) \times m N}\right) .
\end{aligned}
$$

Let $l_{1 i}, l_{2 i}, \ldots, l_{k(i), i}$ be linearly independent columns of the matrix $V_{i}$. Using skeletal decomposition [23] yields $V_{i}=L_{i} \Gamma_{i}$, 
where $L_{i}=\left(l_{1 i}, l_{2 i}, \ldots, l_{k(i), i}\right) \in R_{k(i)}^{(n-q) \times k(i)}, \Gamma_{i} \in R_{k(i)}^{k(i) \times m N}$, and $R_{r}^{k \times l}$ are a set of $k \times l$ matrices that have rank $r$. Since $\widetilde{\Gamma}_{i}=\Gamma_{i} \Gamma_{i}^{T}>0$ is the Gram matrix generated by linearly independent rows of the matrix $\Gamma_{i}$ and $\operatorname{rank}\left(L_{i}\right)=\operatorname{rank}\left(\widetilde{\Gamma}_{i} L_{i}^{T}\right)$ then

$$
\begin{aligned}
& M_{i+1}=L_{i} \widetilde{\Gamma}_{i} L_{i}^{T}, \\
& M_{i+1}^{+}=\left(L_{i} \widetilde{\Gamma}_{i} L_{i}^{T}\right)^{+}=\left(L_{i}^{T}\right)^{+} \widetilde{\Gamma}_{i}^{-1} L_{i}^{+} .
\end{aligned}
$$

Substituting these expressions in (35) and using the representation $L_{i}^{+}=\left(L_{i}^{T} L_{i}\right)^{-1} L_{i}^{T}$, we find

$$
\begin{aligned}
K_{i}^{T} Z_{i+1, t} & =C_{i} R_{i}\left(L_{i}^{T}\right)^{+} \widetilde{\Gamma}_{i}^{-1} L_{i}^{+} L_{i} \widetilde{\Gamma}_{i} L_{i}^{T} M_{t}^{-1} R_{t}^{T} \\
& =C_{i} R_{i} L_{i}\left(L_{i}^{T} L_{i}\right)^{-1} L_{i}^{T} M_{t}^{-1} R_{t}^{T} .
\end{aligned}
$$

It follows from (37) that $R_{i}^{T} C_{i}^{T}=L_{i} \Gamma_{1 i}$, where $\Gamma_{1 i}$ is some rectangular matrix. Substituting this expression in (39) gives

$$
\begin{aligned}
K_{i}^{T} Z_{i+1, t} & =\Gamma_{1 i}^{T} L_{i}^{T} L_{i}\left(L_{i}^{T} L_{i}\right)^{-1} L_{i}^{T} R_{i}^{T} M_{t}^{-1} R_{t}^{T} \\
& =C_{i} R_{i} M_{t}^{-1} R_{t}^{T} .
\end{aligned}
$$

The RHUFIR filter is specified by the following theorem.

Theorem 3. The state of the system

$$
\begin{aligned}
& \widehat{x}_{i+1}=A_{i} \widehat{x}_{i}+K_{i}\left(y_{i}-C_{i} \widehat{x}_{i}\right), \\
& \qquad i \in[t-N, t-1], t \in T_{N}, \\
& \widehat{x}_{t-N}=\widetilde{m}_{t-N}=\left(\left(m_{t-N}\right)^{T}, 0_{1 \times(n-q)}\right)^{T},
\end{aligned}
$$

is the unbiased estimate of the system state (1) for $i \geq t r, t r=$ $\min _{i}\left\{i: M_{i}>0, i=t-N, t-N+1, \ldots\right\}$, where

$$
\begin{aligned}
K_{i} & =A_{i} R_{i}\left(M_{i}+R_{i}^{T} C_{i}^{T} C_{i} R_{i}\right)^{+} R_{i}^{T} C_{i}^{T}, \\
R_{i+1} & =A_{i} R_{i}, \\
R_{t-N} & =\left(e_{q+1}, e_{q+2}, \ldots, e_{n}\right), \\
M_{i+1} & =M_{i}+R_{i}^{T} C_{i}^{T} C_{i} R_{i}, \\
M_{t-N} & =0 .
\end{aligned}
$$

Proof. Put in (2)

$$
\begin{aligned}
Y_{t, i} & =\left(U_{i, t}\right)^{T}=R_{i} M_{i}^{-1} R_{t}^{T} C_{t}^{T}, \\
\Omega_{t, t-N} & =Z_{t-N, t}^{T}\left(e_{q+1}, e_{q+2}, \ldots, e_{n}\right), \quad t \geq t r,
\end{aligned}
$$

where the matric functions are identified in Theorem 1 . This implies the unbiasedness of the estimate at the moment $i \geq t r$ and the relation

$$
\widehat{x}_{i}=\sum_{s=t-N}^{i-1}\left(U_{s, t}\right)^{T} y_{s}+Z_{t-N, i}^{T} \widetilde{m}_{t-N} .
$$

From Lemma 2 it follows that

$$
U_{s, t}=U_{s, t}^{f}=K_{s}^{T} Z_{s+1, t}^{f}
$$

In view of this

$$
\begin{aligned}
\widehat{x}_{i} & =\sum_{s=t-N}^{i-1}\left(U_{s}^{f}\right)^{T} y_{s}+\left(Z_{t-N, t}^{f}\right)^{T} \widetilde{m}_{t-N} \\
& =\sum_{s=t-N}^{i-1} H_{s+1, i}^{T} K_{s} y_{s}+H_{t-N, i}^{T} \widetilde{m}_{t-N},
\end{aligned}
$$

where

$$
\begin{aligned}
& H_{s, i}=\left(A_{s}^{T}-C_{s}^{T} K_{s}^{T}\right) H_{s+1, i}, \\
& H_{i, i}=I_{n},
\end{aligned}
$$

$$
s \leq i .
$$

By (48) we find

$$
\begin{aligned}
\widehat{x}_{i+1}-\widehat{x}_{i}= & K_{i} y_{i}+\sum_{s=i-N}^{i}\left(H_{s+1, i+1}^{T}-H_{s+1, i}^{T}\right) K_{s} y_{s} \\
& +\left(H_{t-N, i+1}^{T}-H_{t-N, i}^{T}\right) \widetilde{m}_{t-N} .
\end{aligned}
$$

Using the identities

$$
\begin{aligned}
H_{s+1, i+1} & =H_{s+1, i} H_{i, i+1}, \\
H_{t-N, i+1} & =H_{t-N, i} H_{i, i+1}, \\
H_{i, i+1} & =A_{i}^{T}-C_{i}^{T} K_{i}^{T},
\end{aligned}
$$

we transform this expression to the form (41)

$$
\begin{aligned}
\widehat{x}_{i+1}-\widehat{x}_{i}= & K_{i} y_{i}+\left(H_{i, i+1}^{T}-I_{n}\right) \sum_{s=t-N}^{i-1} H_{s+1, i}^{T} K_{s} y_{s} \\
& +\left(H_{i, i+1}^{T}-I_{n}\right) H_{t-N, i}^{T} \widetilde{m}_{t-N} \\
= & K_{i} y_{i}+\left(A_{i}-K_{i} C_{i}-I_{n}\right) \\
& \cdot\left[\sum_{s=t-N}^{i-1} H_{s+1, i}^{T} K_{s} y_{s}+H_{t-N, i}^{T} \widetilde{m}_{t-N}\right] \\
= & K_{i} y_{i}+\left(A_{i}-K_{i} C_{i}-I_{n}\right) \widehat{x}_{i} .
\end{aligned}
$$

Putting in (48) $i=t-N$ gives $\widehat{x}_{t-N}=\widetilde{m}_{t-N}$.

Comment 1. The filter is equal to the algorithm from [11] up to the used notation for $q=0$ if to ignore the one-step prediction and to use Theorem 1 to initialize it:

$$
\begin{aligned}
\widehat{x}_{i+1}=A_{i} \widehat{x}_{i}+K_{i}\left(y_{i}-C_{i} \widehat{x}_{i}\right), & \\
& i \in[t-N, t-1], t \in\{2 N, 2 N+1, \ldots\}, \\
\widehat{x}_{N}= & \Phi_{N, 0} M_{N}^{-1} \sum_{i=0}^{N-1} \Phi_{i, 0}^{T} C_{i}^{T} y_{i},
\end{aligned}
$$


where

$$
\begin{aligned}
K_{i} & =A_{i} R_{i}\left(M_{i}+R_{i}^{T} C_{i}^{T} C_{i} R_{i}\right)^{-1} R_{i}^{T} C_{i}^{T}, \\
R_{i+1} & =A_{i} R_{i}, R_{t-N}=I_{n}, \\
M_{i+1} & =M_{i}+R_{i}^{T} C_{i}^{T} C_{i} R_{i}, \\
M_{t-N} & =0 .
\end{aligned}
$$

Consider the system for the state transition matrix of the homogeneous part of the UFIR filter (41)

$$
\begin{aligned}
X_{i+1, s} & =\left(A_{i}-K_{i} C_{i}\right) X_{i, s}=\widetilde{A}_{i} X_{i, s}, \\
X_{s, s} & =I_{n},
\end{aligned}
$$

$$
i \geq s \text {. }
$$

Consequence 1. The following representations are true:

$$
\begin{gathered}
X_{i, s}=\Phi_{i, s}-R_{i} M_{i}^{-1} \sum_{j=s}^{i-1} R_{j}^{T} C_{j}^{T} C_{j} \Phi_{j, s}, \\
\quad i \geq t r, s<i, \\
K_{s}^{T} X_{i, s+1}^{T}=C_{s} R_{s} M_{i}^{-1} R_{i}^{T}, \quad i \geq t r, s<i .
\end{gathered}
$$

Since

$$
\begin{aligned}
& H_{s, i}=Z_{s, i}^{f}=\widetilde{A}_{s}^{T} \widetilde{A}_{s+1}^{T} \ldots \widetilde{A}_{i-1}^{T}, \\
& X_{i, s}=H_{s, i}^{T}=\widetilde{A}_{t-1} \widetilde{A}_{t-2} \cdots \widetilde{A}_{s}
\end{aligned}
$$

the statement follows from (34) and (35).

Consequence 2. Let us show using (56) that the estimate $\widehat{x}_{i}$ is unbiased indeed. Consider the system of equations for the expectation of the estimation error $e_{i}=E\left(x_{i}-\widehat{x}_{i}\right)$

$$
\begin{aligned}
& e_{i+1}=\left(A_{i}-K_{i} C_{i}\right) e_{i}, \\
& e_{t-N}=\left(0_{1 \times q}, x_{q}\right)^{T}, \quad \\
& i \geq t r,
\end{aligned}
$$

where $x_{q}=\left(x_{q+1, t-N}, x_{q+2, t-N}, \ldots, x_{n, t-N}\right)^{T}$ is the arbitrary vector. We find from (56)

$$
\begin{aligned}
e_{i} & =X_{i, t-N} e_{t-N} \\
& =\left(\Phi_{i, t-N}-R_{i} M_{i}^{-1} \sum_{j=t-N}^{i-1} R_{j}^{T} C_{j}^{T} C_{j} \Phi_{j, t-N}\right) R_{t-N} x_{q} \\
& =0, \quad i \geq t r
\end{aligned}
$$

for $q \in\{1,2, \ldots, n-1\}$. Similarly, it is verified that $e_{i}=0$ for $i \geq t r, q=0$, and $e_{t-N}=x_{t-N}$, where $x_{t-N}$ is the arbitrary vector.
Consequence 3. Let the system (1) be time-invariant, $q=0$, and $\operatorname{det} A \neq 0$. Then the representation for the state transition matrix $X_{i, s}$ has the form

$$
X_{i, s}= \begin{cases}0, & s=0, i>t r \\ A^{i-t+N} M_{i}^{-1} M_{s} A^{-s+t-N}, & i>t r, s<i, s \neq 0 .\end{cases}
$$

The statement can be obtained from (56) with the help of elementary calculations.

\section{Comparison of RHUFIR and RHOUFIR Filters}

Let us assume that the following conditions are held.

B1: $w_{t}$ and $\xi_{t}$ are the uncorrelated random processes with zero means and known covariance matrices $E\left(w_{t} w_{t}^{T}\right)=$ $V_{t}, E\left(\xi_{t} \xi_{t}^{T}\right)=\Sigma_{t}, A_{t}, B_{t}, C_{t}, D_{t}$ are known matrices of appropriate dimensions.

B2: Means and covariances are known for arbitrary $q \in$ $\{0,1, \ldots, n-1\}$ components of the state vector at the starting points $x_{t-N}$ of the sliding windows. A priori information on remaining $n-q$ components of $x_{t-N}, t \in T_{N}$ is absent and they are either unknown constants or random variables the statistical characteristics of which are unknown. The value $q=0$ is used further if a priori information about all components of $x_{t-N}$ is absent.

B3: If $q \neq 0$ then without loss of generality it is assumed that the state vector elements are arranged so that means and covariances

$$
\begin{aligned}
m_{i, t-N} & =E\left(x_{i, t-N}\right), \\
\bar{s}_{i j, t-N} & =E\left[\left(x_{i, t-N}-m_{i, t-N}\right)\left(x_{j, t-N}-m_{j, t-N}\right)\right], \\
& i, j=1,2, \ldots, q
\end{aligned}
$$

are known, where $x_{t}=\left(x_{1, t}, \ldots, x_{q, t}, x_{q+1, t}, \ldots, x_{n, t}\right)^{T}, t \in$ $T_{N}=\{N, N+1, \ldots\}$.

B4: If $x_{i, t-N}, i=1,2, \ldots, q$ are random variables then they are uncorrelated with $x_{i, t-N}, i=q+1, q+2, \ldots, n, w_{t}$ and $\xi_{t}$ for $t \in T$.

It is required to find the unbiased linear state estimate of (1) minimizing the criterion $E\left[\left(x_{t}-\widehat{x}_{t}\right)^{T}\left(x_{t}-\widehat{x}_{t}\right)\right]$. This estimate is called the optimal estimate of the system state (1).

Theorem 4 ([8,9]). (1) The optimal estimate of $x_{t}$ (the RHOUFIR filter) is determined by the following relations for $i \geq t r, t r=\min _{i}\left\{i: M_{i}>0, i=t-N, t-N+1, \ldots\right\}$ :

Prediction:

$$
\begin{aligned}
& \widehat{x}_{i+1}^{-}=A_{i} \hat{x}_{i}^{+}, \widehat{x}_{t-N}^{+}=\left(\left(m_{t-N}\right)^{T}, 0_{1 \times(n-q)}\right)^{T}, \\
& \quad i \in[t-N, t-1], t \in T_{N}, \\
& K_{i}=\left[A_{i} S_{i}^{-}+A_{1 i} R_{i}\left(M_{i}+R_{i}^{T} C_{i}^{T} N_{i}^{-1} C_{i} R_{i}\right)^{+} R_{i}^{T}\right] \\
& \cdot C_{i}^{T} N_{i}^{-1},
\end{aligned}
$$




$$
\begin{aligned}
& S_{i}^{-}=A_{i} S_{i}^{+} A_{i}^{T}+B_{i} V_{i} B_{i}^{T}, \\
& S_{t-N}^{+}=\text {block diag }\left(\bar{S}_{t-N}, 0_{(n-q) \times(n-q)}\right) \\
& R_{i+1}=A_{1 i} R_{i}, R_{t-N}=\left(e_{q+1}, \ldots, e_{n}\right) \\
& A_{1 i}=A_{i}-A_{i} S_{i}^{-} C_{i}^{T} N_{i}^{-1} C_{i} \\
& N_{1 s}=C_{s} S_{i}^{-} C_{s}^{T}+D_{i} \Sigma_{i} D_{i}^{T} \\
& M_{i+1}=M_{i}+R_{i}^{T} C_{i}^{T} N_{i}^{-1} C_{i} R_{i} \\
& M_{t-N}=0
\end{aligned}
$$

Correction:

$$
\begin{aligned}
S_{i}^{+} & =\left(I_{n}-S_{i}^{-} C_{i}^{T} N_{i}^{-1} C_{i}\right) S_{i}^{-}, \\
\hat{x}_{i+1}^{+} & =\widehat{x}_{i}^{-}+K_{i}\left(y_{i}-C_{i} \widehat{x}_{i}^{-}\right) .
\end{aligned}
$$

(2) The estimation error covariance matrix for $i \geq t_{t r}$ is given by the expression

$$
P_{i}=S_{i}^{-}+R_{i} M_{i}^{-1} R_{i}^{T} .
$$

Comment 1. Formally, the RHUFIR filter follows from the RHOUFIR filter by specifying $V_{t}=I_{m}, S_{t-N}^{+}=0$, and $\Sigma_{t}=$ 0 . At the same time, the statement of the problem and the derivation of the UFIR filter do not rely on these assumptions.

Comment 2. The relation (71) is the decomposition of the error covariance matrix into two terms. The first one takes into account the known statistical information about initial conditions, process, and measurement noises covariance matrices on the estimate accuracy. The second one additionally reflects the impact of unknown initial conditions at starting points of sliding windows.

Comment 3. Similarly to the covariance matrix, the decomposition is true for the UOFIR filter gain. Indeed, it follows from (64) that $K_{i}=K_{i}^{K F}+K_{i}^{U K F}$, where the term $K_{i}^{K F}=$ $A_{i} S_{i}^{-} C_{i}^{T} N_{i}^{-1}$ coincides with the $\mathrm{KF}$ gain taking in account the known statistical information and the term $K_{i}^{U K F}=$ $A_{1 i} R_{i} M_{i+1}^{+} R_{i}^{T} C_{i}^{T} N_{i}^{-1}$ reflects the additional effect connected with unbiasedness of the RHOUFIR filter.

\section{Receding Horizon FIR Filter with Diffuse Initialization}

Consider an alternative approach to constructing the FIR estimator which is important in view of possible applications. Now, we assume that the condition $\mathrm{B} 1$ from the previous section and the following conditions are met.

$\mathrm{Cl}$ : Means and covariances are known for arbitrary $q \epsilon$ $\{0,1, \ldots, n-1\}$ components of the state vector at the starting points $x_{t-N}$ of the sliding windows.

C2: If $q=0$ then $x_{i, t-N}, i=1,2, \ldots, n$ are treated as random variables with zero mean and covariance matrix proportional to the large parameter $\mu>0$, i.e., $E\left(x_{t-N}\right)=0$,
$E\left(x_{t-N} x_{t-N}^{T}\right)=\mu \widetilde{S}_{t-N}$, where $\widetilde{S}_{t-N}$ is an arbitrary positive definite matrix.

C3: If $q \in\{1,2, \ldots, n-1\}$ then without loss of generality it is assumed that the state vector elements are arranged so that means and covariances

$$
\begin{aligned}
m_{i, t-N} & =E\left(x_{i, t-N}\right), \\
\bar{s}_{i j, t-N} & =E\left[\left(x_{i, t-N}-m_{i, t-N}\right)\left(x_{j, t-N}-m_{j, t-N}\right)\right], \\
& i, j=1,2, \ldots, q
\end{aligned}
$$

are known. A priori information on remaining $n-q$ components of $x_{t-N}, t \in T_{N}$, is absent and they are treated as random variables with zero mean and covariance matrix proportional to the large parameter $\mu>0$, i.e.,

$$
\begin{aligned}
& E\left(x_{i, t-N}\right)=0, \\
& E\left(x_{i, t-N} x_{j, t-N}\right)=\mu \widetilde{s}_{i j, t-N}, \\
& i, j=q+1, q+2, \ldots, n,
\end{aligned}
$$

where $\widetilde{S}_{t-N}=\left\|\widetilde{s}_{i j, t-N}\right\|_{q+1}^{n}>0, \mu>0$ is a large parameter.

C4: The random vector $x_{t-N}$ is not correlated with $w_{t}$ and $\xi_{t}$ for $[t-N, t-1], t \in T_{N}=\{N, N+1, \ldots\}$.

C5: The random variables $x_{i, t-N}$ for $i=1,2, \ldots, q, q \in$ $\{1,2, \ldots, n-1\}$, are uncorrelated with variables $x_{i, t-N}, i=q+$ $1, q+2, \ldots, n$.

It is required to find the limit relations for the $\mathrm{KF}$ as $\mu \longrightarrow$ $\infty$ and to study their properties. In $[8,9]$, it was shown that as $\mu \longrightarrow \infty$ these relations are coincide with (63)-(70).

The conditions $\mathrm{C} 1-\mathrm{C} 5$ formalize the standard approach to the implementation of the KF for large initial uncertainty of the system state. At the same time, it is well known [9, 24] that the large values of $\mu$ can lead to a divergence of the KF. In $[8,9]$, the influence of the large values of $\mu$ on the KF divergence was studied. In particular, let $\delta M_{i}^{+}$be the error connected with calculations of $M_{i}^{+}$. Then

$$
\begin{aligned}
K_{i}= & R_{t} \widetilde{S}_{t-N}\left(I_{n-q}-M_{i}\left(M_{i}^{+}+\delta M_{i}^{+}\right)\right) R_{i}^{T} C_{i}^{T} N_{i}^{-1} \mu \\
& +O(1), \quad \mu \longrightarrow \infty .
\end{aligned}
$$

For $\delta M_{i}^{+} \neq 0$ the matrix $K_{t}$ becomes proportional to the large parameter $\mu$ and so divergence is possible even if the continuity condition of the matrix pseudoinversion finding is performed. Thus, the use of the limiting relations for the filter allows avoiding divergence of the FIR filters with the diffuse initialization.

Note that, for the special case $N=0$, the relations (63)-(70) (the IIR filter) can be used for the state estimation in absence or incompleteness of the statistical information about the initial conditions of (1). Following [8,9], we call them the KF with diffuse initial conditions (DKF).

\section{Application of Receding Horizon FIR Filters to Sea Target Tracking Problem}

7.1. Models of Objects and Measurements. To illustrate the capability of the proposed FIR algorithms, we use the following scenario. With the help of the camera installed on the 


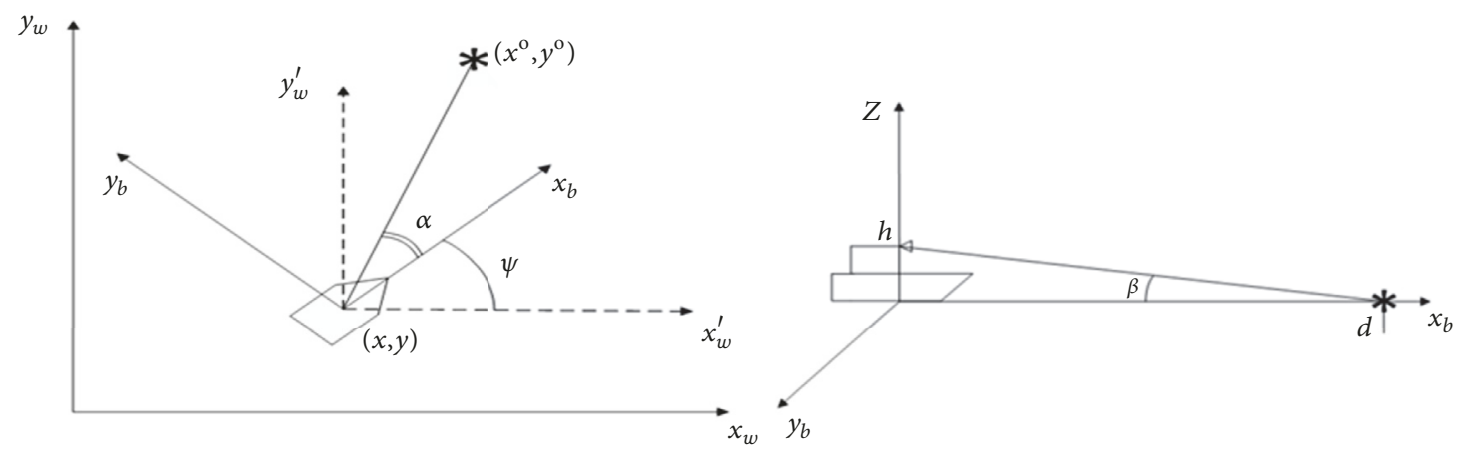

FIGURE 1: Ship and target positions.

ship, the bearing $(\alpha)$, and elevation $(\beta)$ angles to the target (Figure 1) are measured.

The expressions for $\alpha$ and $\beta$ taking into account measurement errors have the following form:

$$
\begin{aligned}
& \alpha_{t}=\operatorname{atan}\left(\frac{-e_{t}^{x} \sin (\psi)+e_{t}^{y} \cos (\psi)}{e_{t}^{x} \cos (\psi)+e_{t}^{y} \sin (\psi)}\right)+\xi_{1 t}, \\
& \beta_{t}=\operatorname{atan}\left(\frac{h_{t}}{\sqrt{\left(e_{t}^{x}\right)^{2}+\left(e_{t}^{y}\right)^{2}}}\right)+\xi_{2 t},
\end{aligned}
$$

where $e_{t}^{x}=x_{t}^{t}-x_{t}^{o}$ and $e_{t}^{y}=y_{t}^{t}-y_{t}^{o}, x_{t}^{t}, y_{t}^{t}$ are the positions of the target, $h_{t}$ is altitude of the camera, $\xi_{1, t}$ and $\xi_{2, t}$ are the centered uncorrelated white noises with the variances $\sigma_{1}^{2}$ and $\sigma_{2}^{2}$, respectively, and atan is the 4-quadrant arctangent.

It is assumed that the variability of $h_{t}$ is caused by a rolling with the dominant frequency and amplitude. More exactly, it is assumed that $h_{t}=h+\delta_{t}, \delta_{t}=p \sin (\omega \Delta t)$, where $h$ is approximately known and $p, \omega$ are unknown values to the observer and $\left|\delta_{t}\right|<<h, \Delta$ is the sampling step. The function $\delta_{t}$ is interpreted as an uncontrolled disturbance in the elevation angle measurement channel.

The ship and the measurements model are described by a nonlinear discrete system of the form

$$
\begin{aligned}
q_{t+1}^{o} & =f^{o}\left(q_{t}^{o}, u_{t}, w_{t}^{o}\right), \\
q_{0}^{o} & =q^{o}, \\
\eta_{t} & =g^{o}\left(q_{t}^{o}\right)+\xi_{t}, \quad t=0,1, \ldots,
\end{aligned}
$$

where $q_{t}^{o} \in R^{n}$ is the state vector, $\eta_{t} \in R^{m}$ is the measured output vector, $w_{t}^{o} \in R^{r}$ and $\xi_{t} \in R^{m}$ are random processes, $u_{t}$ is the control, and $f^{o}(\cdot, \cdot)$ and $g^{o}(\cdot)$ are known vector functions.

The movement of the target to the observer is unknown and we rely on the common approach to describe its behavior consisting in the following $[15,24]$. It is assumed that the target movement can be specified by means of a stochastic system of the form

$$
\begin{aligned}
q_{t+1}^{t} & =f^{t}\left(q_{t}^{t}, w_{t}^{t}\right), \\
q_{0}^{t} & =q^{t},
\end{aligned}
$$

where $q_{t}^{t} \in R^{l}$ is the state vector and $w_{t}^{t} \in R^{d}$ is the random process. A priori information about the initial position of the target is absent and the right-hand parts of the system (79) and the state vector $q_{t}^{t}$ and the random process $w_{t}^{t}$ are chosen using the specifics of the problem under consideration.

Special cases of models (77), (78), and (79) are as follows $[15,24]$.

The ship moves at a constant fixed velocity in accordance with

$$
\begin{aligned}
& x_{t+1}^{o}=x_{t}^{o}+v^{o} \cos (\psi) \Delta, \\
& y_{t+1}^{o}=y_{t}^{o}+v^{o} \sin (\psi) \Delta,
\end{aligned}
$$

where the linear speed $\left(v^{o}\right)$ and the heading angle $(\psi)$ of the ship are known values and $\Delta$ is the sampling period. The coordinates of the ship in the horizontal plane $\left(x_{t}^{o}, y_{t}^{o}\right)$ are measured and the observation models have the form

$$
\begin{aligned}
& z_{3, t}=x_{t}^{o}+\xi_{3 t}, \\
& z_{4, t}=y_{t}^{o}+\xi_{4 t}, \\
& \quad t=0,1, \ldots,
\end{aligned}
$$

where $\xi_{3 t}$ and $\xi_{4 t}$ are the centered uncorrelated white noises with the known variances $\sigma_{3}^{2}$ and $\sigma_{4}^{2}$, respectively, $E x_{0}^{o}=0$, $E y_{0}^{o}=0, \operatorname{cov}\left(x_{0}^{o}\right)=\sigma_{3}^{2}$, and $\operatorname{cov}\left(y_{0}^{o}\right)=\sigma_{4}^{2}$.

The motion of the target is described by

$$
\begin{aligned}
& x_{t+1}^{t}=x_{t}^{t}+\Delta v_{t}^{t, x}+\frac{\Delta^{2}}{2 w_{t}^{x}}, \\
& v_{t+1}^{t, x}=v_{t}^{t, x}+\Delta w_{t}^{x},
\end{aligned}
$$




$$
\begin{aligned}
& y_{t+1}^{t}=y_{t}^{t}+\Delta v_{t}^{t, y}+\frac{\Delta^{2}}{2 w_{t}^{y}}, \\
& v_{t+1}^{t, y}=v_{t}^{t, y}+\Delta w_{t}^{y},
\end{aligned}
$$

$$
t=0,1, \ldots,
$$

where $v_{t}^{t, x}$ and $v_{t}^{t, y}$ are the target velocity projections on coordinates axes $x$ and $y, w_{t}^{x}$, and $w_{t}^{y}$ are the accelerations projections (the centered uncorrelated white noises with the variances $\sigma_{x}^{2}, \sigma_{y}^{2}$, respectively).

7.2. Models of Pseudo Measurements. Let us find expressions for the pseudo measurements model using the relations (75) and (76). The general idea [25] is to represent the nonlinear measurement model $y=h(x)+w$ in the next pseudo linear form $z(y)=H(y) x+w_{z}(x, y)$, where $z(y)$ is the pseudo measurement vector, $H(y)$ is the known function of the true measurements of $y$, and $w_{z}(x, y)$ is the pseudo measurement errors. The $\mathrm{KF}$ is used with $z(y), H(y)$ and $\operatorname{cov}\left[w_{z}(\bar{x}, y)\right]$, where $\bar{x}$ is the predicted state value of $\mathrm{x}$.

Rewrite (75) and (76) in the following form:

$$
\begin{aligned}
& {\left[\tan \left(\alpha_{t}-\xi_{1 t}\right) \cos (\psi)+\sin (\psi)\right] e_{t}^{x}} \\
& \quad+\left[\tan \left(\alpha_{t}-\xi_{1 t}\right) \sin (\psi)-\cos (\psi)\right] e_{t}^{y}=0, \\
& \left(e_{t}^{x}\right)^{2}+\left(e_{t}^{y}\right)^{2}=\left[\frac{h_{t}}{\tan \left(\beta_{t}-\xi_{2 t}\right)}\right]^{2} .
\end{aligned}
$$

We find from (84)

$$
e_{t}^{x}=\frac{\cos (\psi)-\tan \left(\alpha_{t}-\xi_{1 t}\right) \sin (\psi)}{\tan \left(\alpha_{t}-\xi_{1 t}\right) \cos (\psi)+\sin (\psi)} e_{t}^{y}=k e_{t}^{y} .
$$

Substitution of this expression into (85) gives

$$
e_{t}^{y}=\frac{h_{t}}{\tan \left(\beta_{t}-\xi_{2 t}\right)\left(1+k^{2}\right)^{1 / 2}}
$$

and using (86) and (87), we get

$$
e_{t}^{x}=\frac{k h_{t}}{\tan \left(\beta_{t}-\xi_{2 t}\right)\left(1+k^{2}\right)^{1 / 2}} .
$$

As

$$
\begin{aligned}
\left(1+k^{2}\right)^{1 / 2} & =\frac{1}{\sin \left(\alpha_{t}+\psi-\xi_{1 t}\right)}, \\
\frac{k}{\left(1+k^{2}\right)^{1 / 2}} & =\cos \left(\alpha_{t}+\psi-\xi_{1 t}\right)
\end{aligned}
$$

then

$$
\begin{aligned}
& e_{t}^{x}=h_{t} \frac{\cos \left(\alpha_{t}+\psi-\xi_{1 t}\right)}{\tan \left(\beta_{t}-\xi_{2 t}\right)}, \\
& e_{t}^{y}=h_{t} \frac{\sin \left(\alpha_{t}+\psi-\xi_{1 t}\right)}{\tan \left(\beta_{t}-\xi_{2 t}\right)} .
\end{aligned}
$$

Linearizing the right-hand parts of (90) in a neighborhood of the points $\xi_{1 t}=0, \xi_{2 t}=0$, we find

$$
\begin{aligned}
& e_{t}^{x}=h_{t}\left(\frac{\cos \left(\alpha_{t}+\psi\right)}{\tan \left(\beta_{t}\right)}-\zeta_{t}\right), \\
& e_{t}^{y}=h_{t}\left(\frac{\sin \left(\alpha_{t}+\psi\right)}{\tan \left(\beta_{t}\right)}-\eta_{t}\right),
\end{aligned}
$$

where $\zeta_{t}$ and $\eta_{t}$ are correlated random processes defined by expressions

$$
\begin{aligned}
& \varsigma_{t}=\frac{\sin \left(\alpha_{t}+\psi\right)}{\tan \left(\beta_{t}\right)} \xi_{1 t}+\frac{\cos \left(\alpha_{t}+\psi\right)}{\sin ^{2}\left(\beta_{t}\right)} \xi_{2 t}, \\
& \eta_{t}=-\frac{\cos \left(\alpha_{t}+\psi\right)}{\tan \left(\beta_{t}\right)} \xi_{1 t}+\frac{\sin \left(\alpha_{t}+\psi\right)}{\sin ^{2}\left(\beta_{t}\right)} \xi_{2 t} .
\end{aligned}
$$

It follows the relations for the pseudo measurements model

$$
\begin{aligned}
& z_{1 t}\left(\alpha_{t}, \beta_{t}\right)=\frac{\cos \left(\alpha_{t}+\psi\right)}{\tan \left(\beta_{t}\right)}=\frac{\left(-x_{t}^{o}+x_{t}^{t}\right)}{\left(h+\delta_{t}\right)}+\zeta_{t}, \\
& z_{2 t}\left(\alpha_{t}, \beta_{t}\right)=\frac{\sin \left(\alpha_{t}+\psi\right)}{\tan \left(\beta_{t}\right)}=\frac{\left(-y_{t}^{o}+y_{t}^{t}\right)}{\left(h+\delta_{t}\right)}+\eta_{t}
\end{aligned}
$$

where

$$
\begin{gathered}
\sigma_{\varsigma}^{2}=\left[\frac{\sin \left(\alpha_{t}+\psi\right)}{\tan \left(\beta_{t}\right)}\right]^{2} \sigma_{1}^{2}+\left[\frac{\cos \left(\alpha_{t}+\psi\right)}{\sin ^{2}\left(\beta_{t}\right)}\right]^{2} \sigma_{2}^{2}, \\
\sigma_{\eta}^{2}=\left[\frac{\cos \left(\alpha_{t}+\psi\right)}{\tan \left(\beta_{t}\right)}\right]^{2} \sigma_{1}^{2}+\left[\frac{\sin \left(\alpha_{t}+\psi\right)}{\sin ^{2}\left(\beta_{t}\right)}\right]^{2} \sigma_{2}^{2} .
\end{gathered}
$$

Linearizing the right-hand sides of (93) and (94) in a neighborhood of the point $\delta_{t}=0$, we also get

$$
\begin{aligned}
& z_{1 t}\left(\alpha_{t}, \beta_{t}\right)=\frac{\cos \left(\alpha_{t}+\psi\right)}{\tan \left(\beta_{t}\right)}=q_{t}\left(-x_{t}^{o}+x_{t}^{t}\right)+\zeta_{t}, \\
& z_{2 t}\left(\alpha_{t}, \beta_{t}\right)=\frac{\sin \left(\alpha_{t}+\psi\right)}{\tan \left(\beta_{t}\right)}=q_{t}\left(-y_{t}^{o}+y_{t}^{t}\right)+\eta_{t},
\end{aligned}
$$

where $q_{t}=\left(1-\delta_{t} / h\right) / h$.

7.3. Implementations of Filters. The models (80)-(83) are used to demonstrate the proposed FIR algorithms implementations. First of all, note that the filters inputs receive measurements defined by the expressions (75) and (76). Two close implementation schemes are developed. The first one is as follows.

Taking in account that $\delta_{t}$ is an unknown quantity and the relation $\left|\delta_{t}\right|<<h$, the pseudo measurements model has form

$$
\begin{aligned}
& z_{1 t}\left(\alpha_{t}, \beta_{t}\right)=\frac{\cos \left(\alpha_{t}+\psi\right)}{\tan \left(\beta_{t}\right)}=\frac{\left(-x_{t}^{o}+x_{t}^{t}\right)}{h}+\zeta_{t}, \\
& z_{2 t}\left(\alpha_{t}, \beta_{t}\right)=\frac{\sin \left(\alpha_{t}+\psi\right)}{\tan \left(\beta_{t}\right)}=\frac{\left(-y_{t}^{o}+y_{t}^{t}\right)}{h}+\eta_{t} .
\end{aligned}
$$


In accordance with the relations (80)-(83), (99), and (100), the following linear system to construct the RHOUFIR filter and the DKF is used:

$$
\begin{aligned}
x_{t+1} & =A x_{t}+w_{t}+f_{t}, \\
y_{t} & =C x_{t}+\mu_{t},
\end{aligned}
$$

where

$$
\begin{aligned}
& x_{t}=\left(x_{t}^{o}, y_{t}^{o}, x_{t}^{t}, v_{t}^{t, x}, x_{t}^{t}, v_{t}^{t, y}\right)^{T}, \\
& f_{t}=\left(v^{o} \cos (\psi) \Delta, v^{o} \sin (\psi) \Delta, 0,0,0,0\right)^{T}, \\
& w_{t}=\left(0,0, \frac{\Delta^{2}}{2 w_{t}^{x}}, \Delta w_{t}^{x}, \frac{\Delta^{2}}{2 w_{t}^{y}}, \Delta w_{t}^{y}\right)^{T}, \\
& \mu_{t}=\left(\xi_{3 t}, \xi_{4 t}, \zeta_{t}, \eta_{t}\right)^{T} \text {, } \\
& A=\left[\begin{array}{llllll}
1 & 0 & 0 & 0 & 0 & 0 \\
0 & 1 & 0 & 0 & 0 & 0 \\
0 & 0 & 1 & \Delta & 0 & 0 \\
0 & 0 & 0 & 1 & 0 & 0 \\
0 & 0 & 0 & 0 & 1 & \Delta \\
0 & 0 & 0 & 0 & 0 & \Delta
\end{array}\right], \\
& C=\left[\begin{array}{cccccc}
1 & 0 & 0 & 0 & 0 & 0 \\
0 & 1 & 0 & 0 & 0 & 0 \\
-1 / h & 0 & -1 / h & 0 & 0 & 0 \\
0 & -1 / h & 0 & 0 & -1 / h & 0
\end{array}\right] \text {, } \\
& E x_{i, t-N}^{o}=0 \text {, } \\
& \operatorname{cov}\left(x_{i, t-N}^{o}\right)=\sigma_{i}^{2}, \\
& i=3,4 \text {, } \\
& E w_{t}^{x}=0, \\
& \operatorname{cov}\left(w_{t}^{x}\right)=\sigma_{x}^{2}, \\
& E w_{t}^{y}=0, \\
& \operatorname{cov}\left(w_{t}^{y}\right)=\sigma_{y}^{2}, \\
& q=2 .
\end{aligned}
$$

It is easy to verify that the pair $\{A, C\}$ is not observable but the condition (4) is fulfilled.

As the RHUFIR filter does not depend on statistics noises we get systems of the form (101) with $q=1$, where

$$
\begin{aligned}
& x_{t}=\left(x_{t}^{o}, x_{t}^{t}, v_{t}^{t, x}\right)^{T}, \\
& f_{t}=\left(v^{o} \cos (\psi) \Delta\right)^{T},
\end{aligned}
$$

$$
\begin{aligned}
& w_{t}=\left(0, \frac{\Delta^{2}}{2 w_{t}^{x}}, \Delta w_{t}^{x}\right)^{T}, \\
& \mu_{t}=\left(\xi_{3 t}, \zeta_{t}\right)^{T}, \\
& x_{t}=\left(y_{t}^{o}, y_{t}^{t}, v_{t}^{t, y}\right)^{T} \text {, } \\
& f_{t}=\left(v^{o} \sin (\psi) \Delta\right)^{T} \text {, } \\
& w_{t}=\left(0, \frac{\Delta^{2}}{2 w_{t}^{x}}, \Delta w_{t}^{x}\right)^{T} \text {, } \\
& \mu_{t}=\left(\xi_{4 t}, \eta_{t}\right)^{T} \text {, } \\
& A=\left[\begin{array}{lll}
1 & 0 & 0 \\
0 & 1 & \Delta \\
0 & 0 & 1
\end{array}\right], \\
& C=\left[\begin{array}{ccc}
1 & 0 & 0 \\
0 & 1 / h & 0
\end{array}\right] \text {, }
\end{aligned}
$$

The second implementation scheme of the RHUFIR filter is determined by the pseudo measurements model:

$$
\begin{aligned}
z_{1 t}\left(\alpha_{t}, \beta_{t}\right) & =\frac{\cos \left(\alpha_{t}+\psi\right)}{\tan \left(\beta_{t}\right)}+\frac{\left(x_{0}^{o}+v^{o} \cos (\psi) \Delta t\right)}{h} \\
& =\frac{x_{t}^{t}}{h}+\zeta_{t}+\xi_{3 t}, \\
z_{2 t}\left(\alpha_{t}, \beta_{t}\right) & =\frac{\sin \left(\alpha_{t}+\psi\right)}{\tan \left(\beta_{t}\right)}+\frac{\left(y_{0}^{o}+v^{o} \sin (\psi) \Delta t\right)}{h} \\
& =\frac{y_{t}^{t}}{h}+\eta_{t}+\xi_{4 t}
\end{aligned}
$$

and the system (101), where

$$
\begin{aligned}
& x_{t}=\left(x_{t}^{t}, v_{t}^{t, x}\right)^{T}, \\
& f_{t}=0, \\
& w_{t}=\left(\frac{\Delta^{2}}{2 w_{t}^{x}}, \Delta w_{t}^{x}\right)^{T}, \\
& \mu_{t}=\zeta_{t}+\xi_{3 t}, \\
& y_{t}=\left(y_{t}^{t}, v_{t}^{t, y}\right)^{T}, \\
& f_{t}=0, \\
& w_{t}=\left(\frac{\Delta^{2}}{2 w^{y}}, \Delta w_{t}^{y}\right)^{T},
\end{aligned}
$$


TABLE 1: Input data for the simulation.

\begin{tabular}{ll}
$\begin{array}{l}\text { Components } \\
\text { of the system }\end{array}$ & Parameters \\
\hline \multirow{2}{*}{ Camera } & Full $\mathrm{HD}(1920 \times 1080), \alpha=70^{0}$ (angle of view), $\sigma_{1}=0.88^{\prime}$, \\
& $\sigma_{2}=1.56^{\prime}, h=3 \mathrm{~m}, \omega=2 \pi / T, T=10 \mathrm{~s}, p=0.3 \mathrm{~m}$ \\
\hline \multirow{2}{*}{ Ship } & $\begin{array}{l}x_{0}^{o}=y_{0}^{o}=0 \mathrm{~m}, \sigma_{3}=\sigma_{4}=1 \mathrm{~m}, v^{o, x}=2.55 \mathrm{~m} / \mathrm{s}, v^{o, y}=4.33 \\
\mathrm{~m} / \mathrm{s}, \psi=60^{0}, \Delta=0.3 \mathrm{~s}\end{array}$ \\
\hline \multirow{2}{*}{ Target } & $\begin{array}{l}x_{0}^{t}=400 \mathrm{~m}, y_{0}^{t}=600 \mathrm{~m}, v_{0}^{t, y}=3.12 \mathrm{~m} / \mathrm{s}, v_{0}^{t, x}=2.98 \mathrm{~m} / \mathrm{s}, \\
\sigma_{x}=\sigma_{y}=0.1 \mathrm{~m} / \mathrm{s}^{2}, \Delta=0.3 \mathrm{~s}, \psi=60^{0}\end{array}$ \\
\hline
\end{tabular}
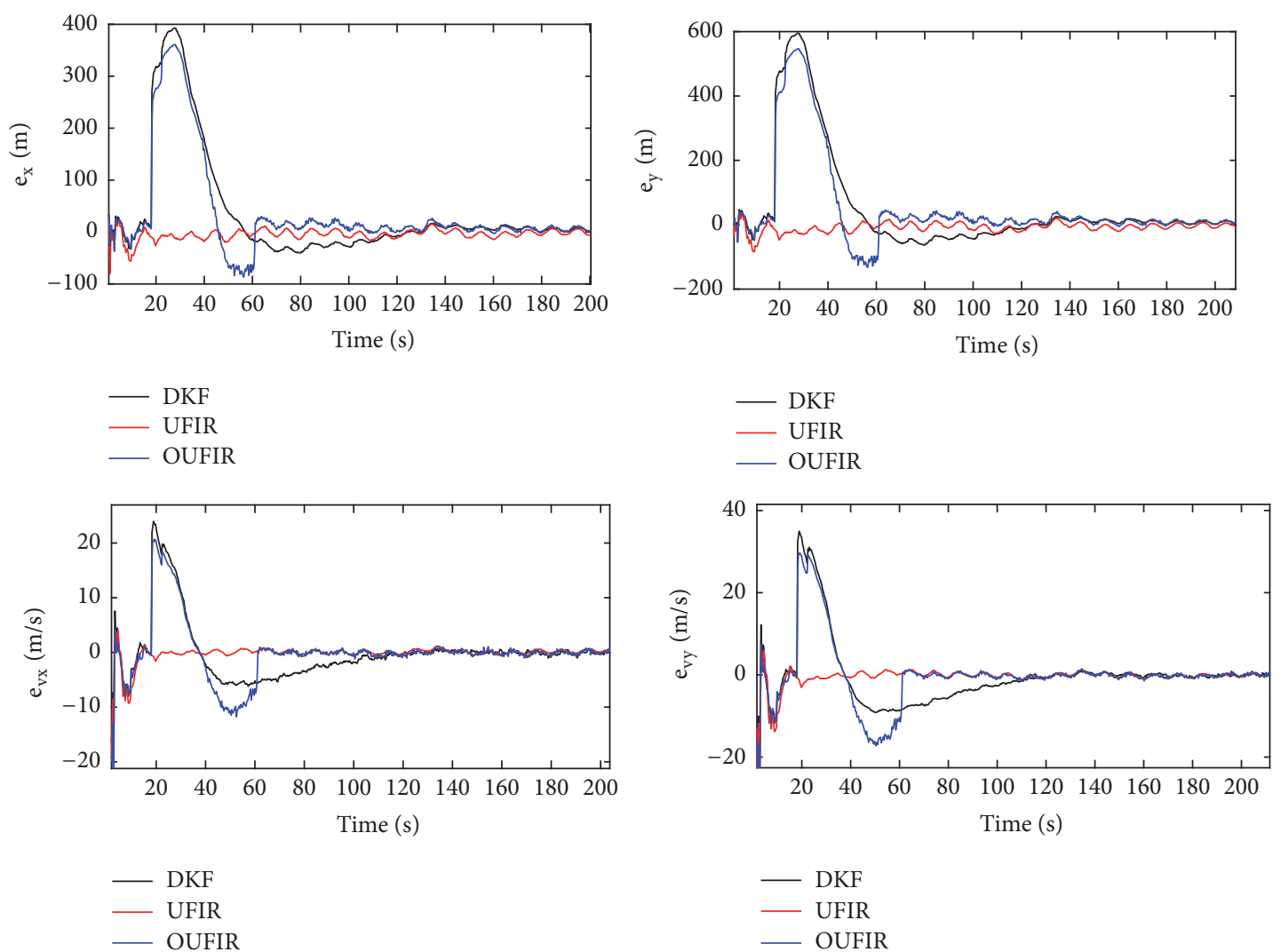

FIGURE 2: Position and velocity estimation errors caused by the signal temporary uncertainty.

$$
\begin{aligned}
\mu_{t} & =\eta_{t}+\xi_{4 t}, \\
A & =\left\{\begin{array}{ll}
1 & \Delta \\
0 & 1
\end{array}\right\}, \\
C & =\left[\begin{array}{ll}
1 / h & 0
\end{array}\right], \\
q & =0 .
\end{aligned}
$$

7.4. Simulation. In this subsection, the behaviors of the DKF, the RHUFIR, and RHOUFIR filters, under the joint action of uncontrolled perturbations, are compared: rolling $\left(\delta_{t}\right)$, impulse perturbations of the bearing angle, and the target acceleration.

Input data for the simulation are listed in Table 1 . We set $N=130$ (a heuristic choice) for the RHUFIR and RHOUFIR filters and $N=0$ for the DKF (the IIR filter).

First, we compare the joint action effect of the rolling and the signal temporary uncertainty on position and velocity estimation errors of the DKF, the RHUFIR and RHOUFIR filters. We set $\alpha_{t}+\lambda_{t}$, where $\lambda_{t}=1^{o}$ when $60 \leq t \leq 75 \mathrm{~s}$ and $\lambda_{t}=0$ otherwise. Estimation errors $\left(e_{x}=x_{t}^{t}-x_{t}^{o}\right.$, $e_{y}=y_{t}^{t}-y_{t}^{o}, e_{v x}=v_{t}^{t, x}-v_{t}^{o, x}$, and $\left.e_{v y}=v_{t}^{t, y}-v_{t}^{o, y}\right)$ are shown in Figure 2. It is seen that the RHUFIR filter provides much more robust estimates compared with the 

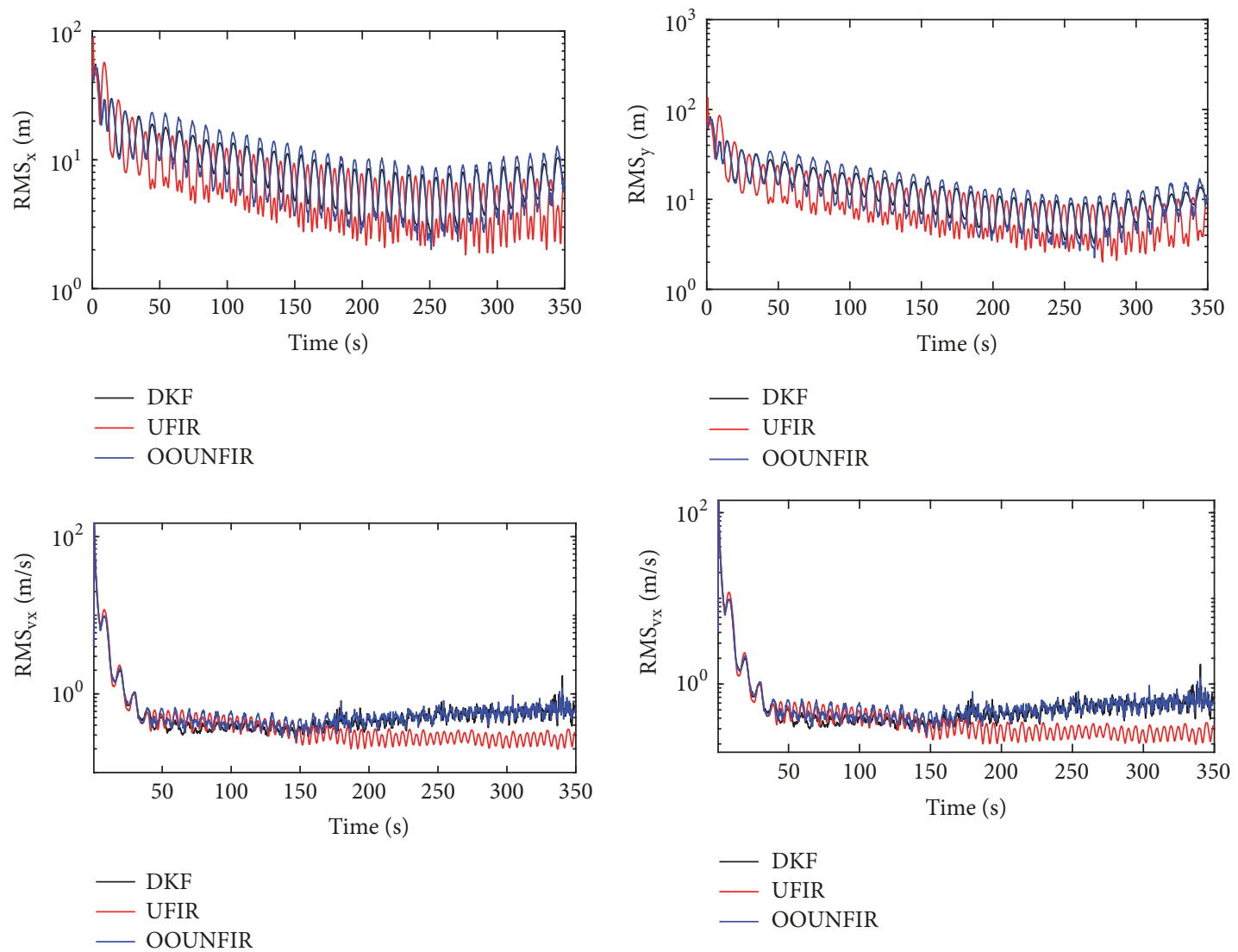

FIGURE 3: RMS position and velocity errors.

DKF and the RHOUFIR filter and the RHOUFIR filter superiors the DKF. This is due to the following reasons. Firstly, the RHUFIR gain does not depend on the bearing $\left(\alpha_{t}\right)$ and elevation $\left(\beta_{t}\right)$ angles. Secondly, the matrix functions $M_{i}, N_{i}$, and $A_{i} S_{i}^{-}+A_{1 i} R_{i} M_{i+1}^{+}$in the expression (64) of the RHOUFIR filter gain are ill-conditioned with the condition number more than $10^{5}$. This may be a consequence of the weak observability of the system with angular observations $[17,18]$. At the same time, it seems that the RHOUFIR filter and the DKF characteristics can be improved by square root implementation.

Compare the joint effect in the inaccurate setting of the target noise statistics and the rolling on position and velocity estimation errors of the RHUFIR and RHOUFIR filters and the DKF. We set $\sigma_{x}=\sigma_{y}=0.13 \mathrm{~m} / \mathrm{s}^{2}$ for $60 \leq t \leq 150 \mathrm{~s}$ and $\sigma_{x}=\sigma_{y}=0.1 \mathrm{~m} / \mathrm{s}^{2}$ otherwise. The Root Mean Square (RMS) position and velocity errors for 100 Monte Carlo runs are shown in Figure 3. It is seen that the RHUFIR filter provides much more robust estimates compared with the DKF and the RHOUFIR filter. The dependencies of position and velocity estimation errors of the RHUFIR filter on the minimum distance $\left(D_{\min }\right)$ between the ship and the target for Monte Carlo runs are shown in Figure 4 . It is seen that $e_{x}$ and $e_{y}$ do not exceed $11 \mathrm{~m}$ and $e_{v x}$ and $e_{v y}-0.8 \mathrm{~m} / \mathrm{s}$ if $D_{\min } \leq$ $300 \mathrm{~m}$.

\section{Conclusions}

The main contributions of this paper are as follows. First, new receding horizon unbiased FIR filters for linear discrete state-space models ignoring the noise statistics of the process and measurements were proposed. The possibility to use only known means of any state vector components at starting points of sliding windows is believed to be one of these filters' vantage points. It allows us to take into account priory statistical information (on average) about random biases, trends, and specified movements of the system. Second, the proposed RHUFIR filter has a Kalman-like form, and it does not require a training cycle in a batch form and has significantly lower complexity compared to the RHOUFIR one. Third, the RHUFIR filter can provide more robust estimation of the position and speed of objects on the sea surface when exposed to uncontrolled disturbances in comparison with the RHOUFIR filter and the DFK (IIR filter). This effect is achieved by the structure of the RHUFIR fitter, the averaging, and the careful selection of the horizon length.

\section{Data Availability}

The data used to support the findings of this study are included within the article. More precisely, all the necessary data for the simulation are given in Table 1. 

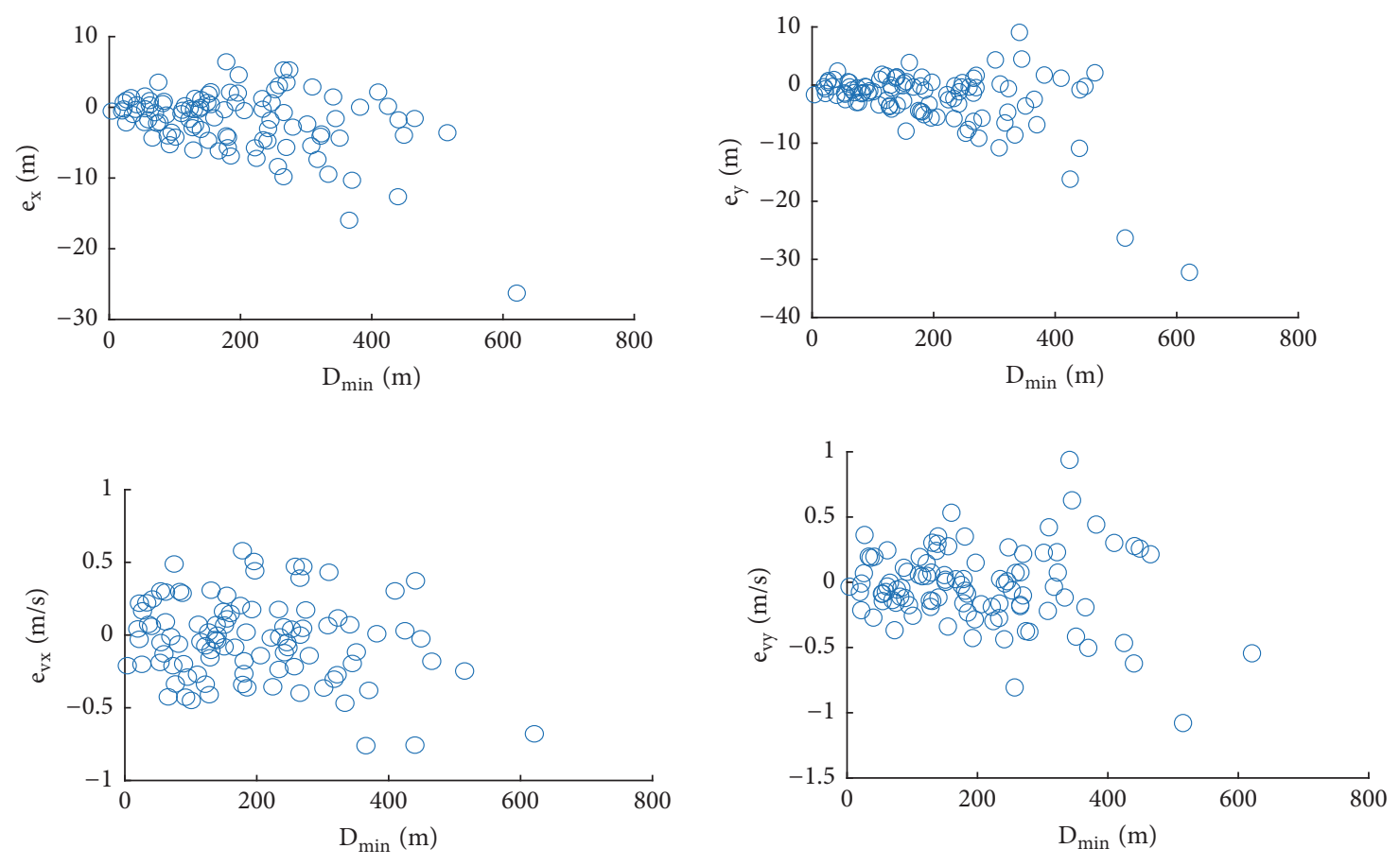

FIGURE 4: Dependencies of position and velocity estimation errors on the minimum distance.

\section{Conflicts of Interest}

The author declares that there are no conflicts of interest regarding the publication of this paper.

\section{Acknowledgments}

Studies were supported by the Ministry of Education and Science of the Russian Federation (Project RFMEFI57818X0264).

\section{References}

[1] A. H. Jazwinski, Stochastic Processes and Filtering Theory, Elsevier, New York, USA, 1970.

[2] W. H. Kwon, P. S. Kim, and P. Park, "A receding horizon Kalman FIR filter for discrete time-invariant systems," IEEE Transactions on Automatic Control, vol. 44, no. 9, pp. 1787-1791, 1999.

[3] Y. S. Shmaliy and O. Ibarra-Manzano, "Time-variant linear optimal finite impulse response estimator for discrete statespace models," International Journal of Adaptive Control and Signal Processing, vol. 26, no. 2, pp. 95-104, 2012.

[4] A. H. Jazwinski, "Limited memory optimal filtering," IEEE Transactions on Automatic Control, vol. 13, no. 5, pp. 558-563, 1968.

[5] W. H. Kwon, P. S. Kim, and S. H. Han, "A receding horizon unbiased FIR filter for discrete-time state space models," Automatica, vol. 38, no. 3, pp. 545-551, 2002.

[6] O. K. Kwon, W. H. Kwon, and K. S. Lee, "FIR filters and recursive forms for discrete-time state-space models," Automatica, vol. 25, no. 5, pp. 715-728, 1989.
[7] P.-S. Kim, "An alternative FIR filter for state estimation in discrete-time systems," Digital Signal Processing, vol. 20, no. 3, pp. 935-943, 2010.

[8] B. A. Skorohod, "Diffuse Initialization of Kalman Filter," Journal of Automation and Information Sciences, vol. 43, no. 4, pp. 20 34, 2011.

[9] B. Skorohod, "Diffuse Algorithms for Neural and Neuro-Fuzzy Networks," in With Applications in Control Engineering and Signal Processing, p. 220, Elsevier, United Kingdom, 2017.

[10] Y. S. Shmaliy, "Unbiased FIR filtering of discrete-time polynomial state-space models," IEEE Transactions on Signal Processing, vol. 57, no. 4, pp. 1241-1249, 2009.

[11] Y. S. Shmaliy, "An iterative Kalman-like algorithm ignoring noise and initial conditions," IEEE Transactions on Signal Processing, vol. 59, no. 6, pp. 2465-2473, 2011.

[12] Y. S. Shmaliy and D. Simon, "Iterative unbiased FIR state estimation: a review of algorithms," EURASIP Journal on Advances in Signal Processing, vol. 2013, article 113, 16 pages, 2013.

[13] F. Ramirez-Echeverria, A. Sarr, and Y. Shmaliy, "Optimal memory for discrete-time FIR filters in state-space," IEEE Transactions on Signal Processing, vol. 62, no. 3, pp. 557-561, 2014.

[14] Z. Yang, X. Li, H. Wang, and R. Fa, "Knowledge-aided STAP with sparse-recovery by exploiting spatio-temporal sparsity," IET Signal Processing, vol. 10, no. 2, pp. 150-161, 2016.

[15] Y. Bar-Shalom, X. Li, and T. Kirubarajan, Estimation with Applications to Tracking and Navigation, John Wiley \& Sons, New York, NY, USA, 2001

[16] B. F. La Scala, M. Mallick, S. Arulampalam, O. E. Drummond, and R. D. Teichgraeber, "Differential geometry measures of nonlinearity for filtering with nonlinear dynamic and linear measurement models," in Proceedings of the Optical Engineering + Applications, San Diego, CA, 2007. 
[17] P. Shar and X. R. Li, "Practical approach to observability of bearings-only target tracking," in Proceedings of the 1999 Signal and Data Processing of Small Targets, pp. 514-520, July 1999.

[18] M. Ferdowsi, "Observability conditions for target states with bearing-only measurements in three-dimensional case," in Proceedings of the 2006 IEEE International Conference on Control Applications, pp. 1444-1449, Munich, Germany, October 2006.

[19] B. La Scala and M. R. Morelande, "Scala Mark Morelande An analysis of the single sensor bearings-only tracking problem," in Proceedings of the 11th International Conference on Information Fusion, vol. 30, pp. 525-530, 2008.

[20] V. J. Aidala, "Kalman Filter Behavior in Bearings-Only Tracking Applications," IEEE Transactions on Aerospace and Electronic Systems, vol. 15, no. 1, pp. 29-39, 1979.

[21] X. Lin, T. Kirubarajan, Y. Bar-Shalom, and S. Maskell, "Comparison of EKF, pseudomeasurement and particle filters for a bearing-only target tracking problem," in Proceedings of the Signal and Data Processing of Small Targets, vol. 4728 of Proceedings of SPIE, pp. 240-250, April 2002.

[22] G. Golub and V. Pereyra, "Separable nonlinear least squares: the variable projection method and its applications," Inverse Problems, vol. 19, no. 2, pp. R1-R26, 2003.

[23] F. R. Gantmaher, "Matrix Theory," M: Fizmathlit, Moscov, 560 pages, 2004.

[24] A. C. Harvey and T. Proietti, Eds., Readings in unobserved components models, Advanced Texts in Econometrics, Oxford University Press, Oxford, 2005.

[25] X.-R. Li and V. P. Jilkov, "Survey of maneuvering target tracking: dynamic models," in Proceedings of the Signal and Data Processing of Small Targets, vol. 4048 of Proceedings of SPIE, pp. 1-24, Orlando, Fla, USA, April 2000. 


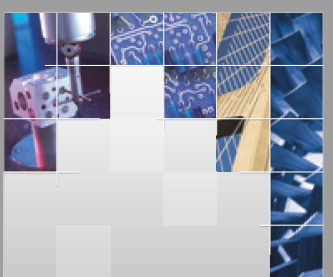

\section{Enfincering}
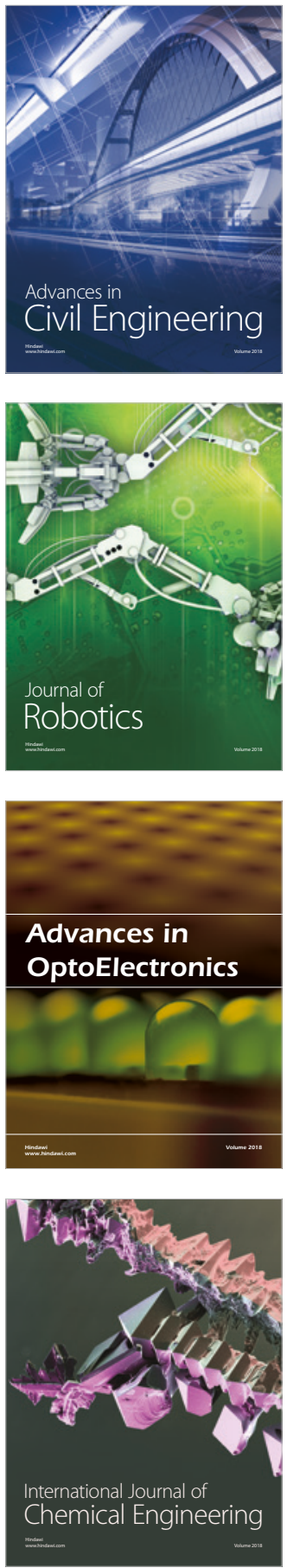

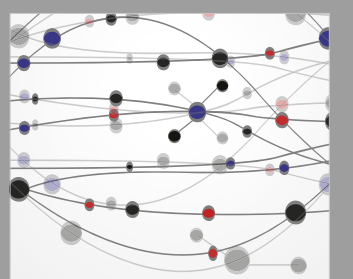

\section{Rotating \\ Machinery}

The Scientific World Journal

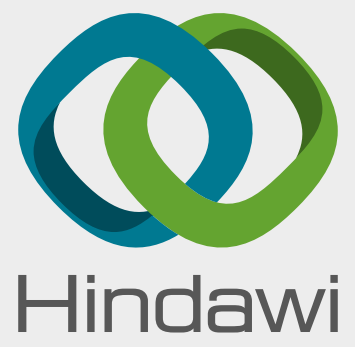

Submit your manuscripts at

www.hindawi.com
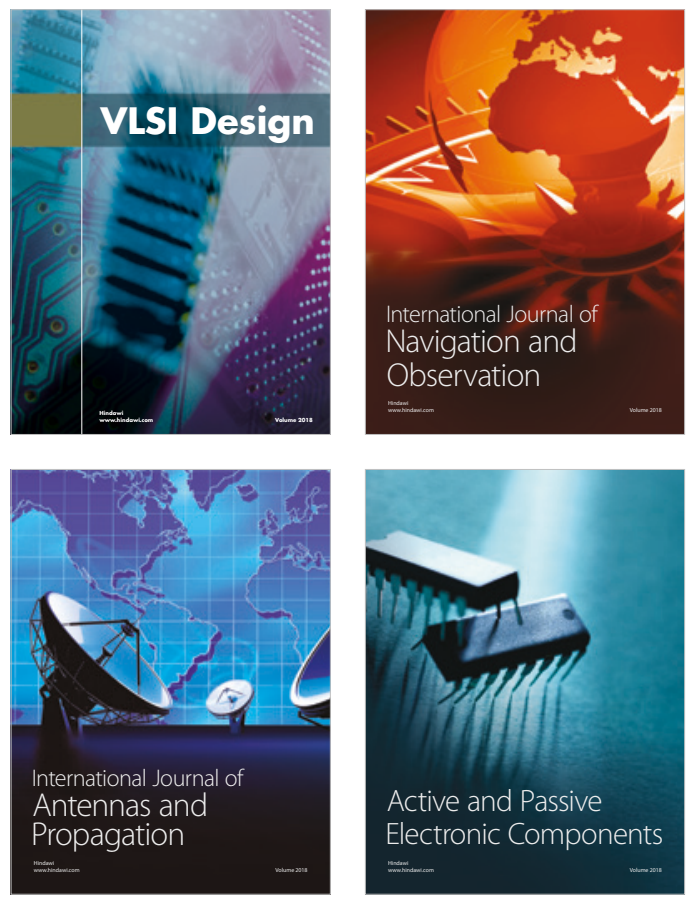
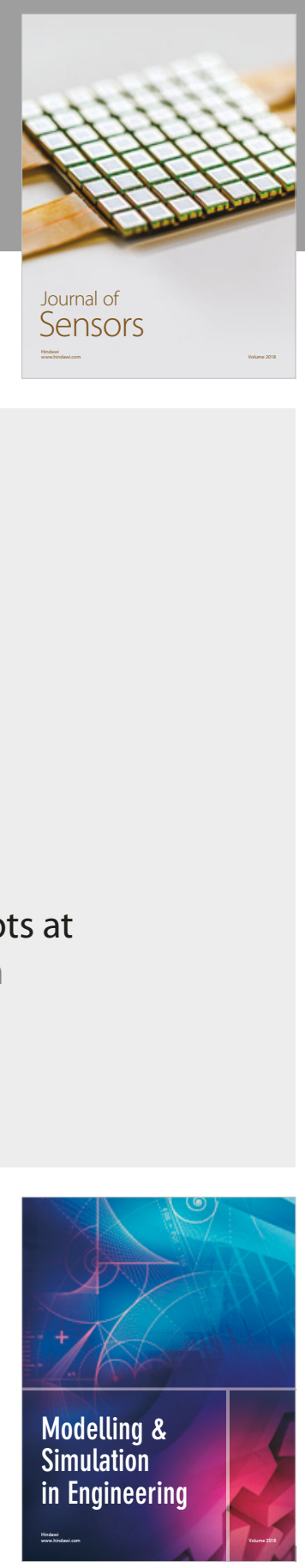

\section{Advances \\ Multimedia}
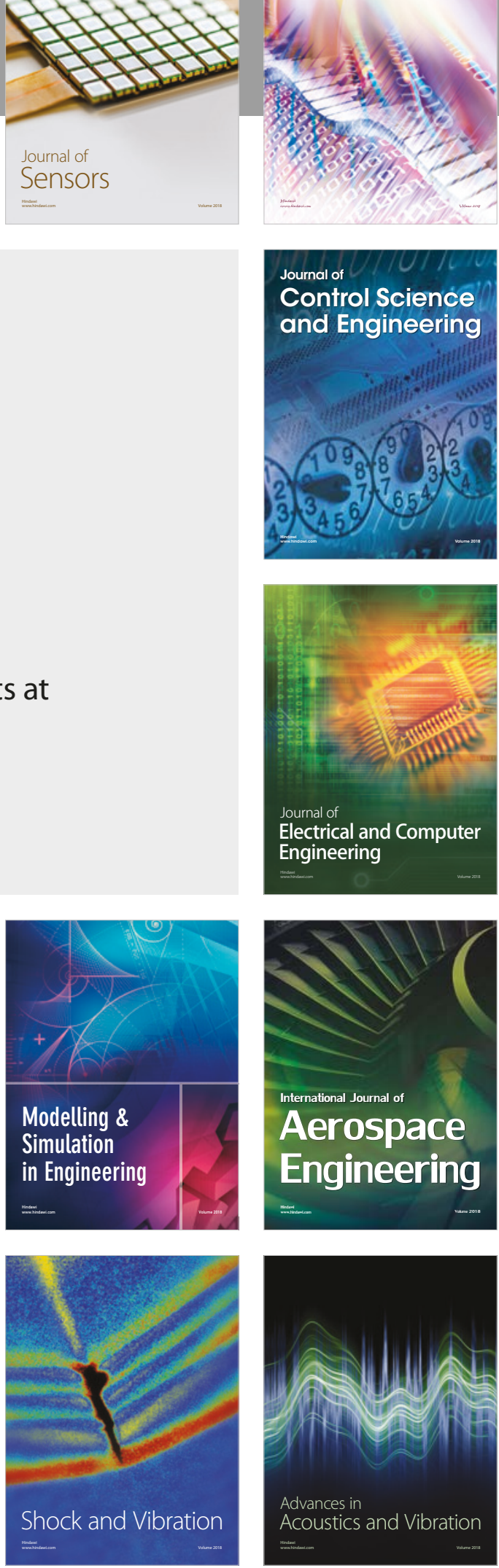